\title{
EFICIÊNCIA E EFEITO RESIDUAL DE INSETICIDAS APLICADOS EM DIFERENTES SUPERFÍCIES PARA O CONTROLE DE BLATTELLA GERMANICA (LINNAEUS, 1767) (DICTYOPTERA: BLATTELLIDAE)
}

\author{
R.S. Parreira, M.C. Ferreira, N.M. Martinelli, I.C. Silva, W.C. Pazini \\ Universidade Estadual Paulista, Faculdade de Ciências Agrárias e Veterinárias, Departamento de Fitossanidade, \\ Via de Acesso Prof. Paulo Donato Castellane, s/nº, CEP 14884-900, Jaboticabal, SP, Brasil. E-mail: wpazini@ \\ fcav.unesp.br
}

\section{RESUMO}

Objetivou-se avaliar eficiência e efeito residual de inseticidas aplicados sobre azulejo (face lisa) e ardósia (rugosa) para o controle de Blattella germanica. Realizou-se o experimento no Departamento de Fitossanidade, UNESP-Jaboticabal, SP (de 3-5 a 2-8-2007). A população suscetível ao controle químico foi criada em sala não climatizada. Os tratamentos utilizados foram (dosagens em produtos comerciais): lambdacialotrina (Demand 2,5 CS) $10 \mathrm{~mL} / \mathrm{L}\left(12,5 \mathrm{mg} / \mathrm{m}^{2}\right)$; lambdacialotrina (Icon 5,0 CE) $5 \mathrm{~mL} / \mathrm{L}\left(12,5 \mathrm{mg} / \mathrm{m}^{2}\right)$; deltametrina (Deltagard WG) 2 saches $/ 10 \mathrm{~L}\left(12,5 \mathrm{mg} / \mathrm{m}^{2}\right)$; lambdacialotrina (Zeon 50CS) $5 \mathrm{~mL} / \mathrm{L}\left(12,5 \mathrm{mg} / \mathrm{m}^{2}\right)$; deltametrina (Deltek CS) $5 \mathrm{~mL} / \mathrm{L}$ (6,25 mg/ $\left.\mathrm{m}^{2}\right)$; Diclorvos (DDVP 1000CE) $5 \mathrm{~mL} / \mathrm{L}\left(250 \mathrm{mg} / \mathrm{m}^{2}\right)$ e testemunha. O delineamento experimental utilizado foi blocos casualizados, com quatro repetições. Os inseticidas foram aplicados com pulverizador pressurizado (350 kPa) e volume de $40 \mathrm{~mL} / \mathrm{m}^{2}$. Uma hora após a aplicação, liberaramse 10 baratas adultas para caminhar sobre a área tratada. Após 15 minutos foram retiradas e colocadas em potes para avaliações $(0,1,2,4,24,48$ e 72 horas após a exposição). Placas tratadas foram mantidas em prateleiras para avaliar o efeito residual dos produtos 30, 60 e 90 dias após aplicação. Os dados foram submetidos à análise de variância e teste Tukey. Concluiu-se que no dia da aplicação dos inseticidas não ocorre diferença entre as superfícies no controle de B. germanica. O efeito residual dos inseticidas foi maior na superfície lisa (azulejo) do que na superfície rugosa (ardósia). Os inseticidas lambdacialotrina e deltametrina (WG) proporcionaram excelente controle de B. germanica, até 90 dias após a aplicação.

PALAVRAS-CHAVE: Barata alemã, manejo de pragas urbanas, tratamento domissanitário.

\section{ABSTRACT}

EFFICIENCY AND RESIDUAL EFFECT OF INSECTICIDES APPLIED ON DIFFERENT SURFACESFOR THE CONTROL OF BLATTELLA GERMANICA (LINNAEUS, 1767) (DICTYOPTERA: BLATTELLIDAE). The aim of the present study was to evaluate the efficiency and residual effect of insecticides applied on tiles and slate for the control of Blattella germanica. The experiment was carried out in the Departamento de Fitossanidade, UNESP Jaboticabal, SP, Brazil, (May 3 to August $5,2007)$. The population susceptible to chemical control was grown in a nonclimatized room. The treatments were lambda-cyhalothrin (Demand 2,5CS) $10 \mathrm{~mL} / \mathrm{L}\left(12,5 \mathrm{mg} / \mathrm{m}^{2}\right)$; lambda-cyhalothrin (Icon 5,0 CE) $5 \mathrm{~mL} / \mathrm{L} \mathrm{(12,5} \mathrm{mg/m²);} \mathrm{deltamethrin} \mathrm{(Deltagard} \mathrm{WG)} 2$ sachets $/ 10 \mathrm{~L}\left(12,5 \mathrm{mg} / \mathrm{m}^{2}\right)$; lambda-cyhalothrin (Zeon 50CS) $5 \mathrm{~mL} / \mathrm{L}\left(12,5 \mathrm{mg} / \mathrm{m}^{2}\right)$; deltamethrin (Deltek CS) $5 \mathrm{~mL} / \mathrm{L}$ (6,25 mg/ $\left.\mathrm{m}^{2}\right)$; dichlorvos (DDVP 1000CE) $5 \mathrm{~mL} / \mathrm{L}\left(250 \mathrm{mg} / \mathrm{m}^{2}\right)$ and a control plot. The experimental design consisted of randomized blocks with four replicates. Insecticides were sprayed with a pressurized sprayer $(350 \mathrm{kPa})$ at a volume of $40 \mathrm{~mL} / \mathrm{m}^{2}$. After $1 \mathrm{~h}, 10$ adult German cockroaches were plotted over the treated area and stayed there for $15 \mathrm{~min}$. They were then transferred to pots for evaluations $(0,1,2,4,24,48$ and $72 \mathrm{~h}$ after exposition). Treated plates were kept on shelves to evaluate the residual effect of products 30,60 and 90 days after application. Data was submitted to analysis of variance and Tukey test. The efficiency was calculated. It was concluded that on the application of insecticides there is no difference between the surfaces in the control of B. germanica. The residual effect was greater on a tiled surface in relation to slate. The insecticides lambda-cyhalothrin and deltamethrin (WG) resulted in sufficient control up to 90 days after the spraying.

KEY WORDS: German cockroach, urban pest management, urban pest control. 


\section{INTRODUÇÃO}

Dentre as principais espécies de artrópodes que se estabeleceram no ambiente urbano, a barata alemã Blattella germanica (Linnaeus, 1767) (Dictyopera: Blattellidae) destaca-se pelo incômodo que provoca pela sua presença. Porém, das cerca de 4.000 espécies de baratas conhecidas no mundo, poucas são consideradas pragas. O elevado potencial reprodutivo, adaptação a ambientes diversos, hábito onívoro, necrofagia e coprofagia são as principais características adaptativas, responsáveis pelo sucesso no convívio com o homem (CORNwELL, 1968).

As baratas permaneceram basicamente inalteradas em relação aos hábitos e forma corpórea nos últimos 300 milhões de anos (GALlo et al., 2002). Transportam patógenos que podem permanecer viáveis em seu tubo digestivo, tegumento e excrementos, durante dias ou semanas. Esses patógenos podem ser transmitidos através de regurgitação dos alimentos, contato ou depósito dos excrementos das baratas. O comportamento das espécies domésticas, de alternarem habitats durante o dia e a noite, as transforma em perigosos agentes de contaminação. Durante o dia repousam em ambientes escuros, úmidos equentes. Ànoite exploram ativamente locais de manipulação e depósito de alimentos (Pérez, 1989).

Diversos métodos de controle têm sido propostos e pesquisados dentro da filosofia do manejo integrado de pragas, destacando-se as iscas (Ross, 1998), armadilhas (Appel, 1995; SMITH II et al., 1997) e os inseticidas (BRANESS, 1990; BRANESS et al., 1991; El-Awami; Dent, 1995; KaAKeH et al., 1997).

O controle da barata alemã tem sido realizado principalmente com inseticidas, e a seleção de populações resistentes aos produtos utilizados é um dos grandes entraves no seu controle, principalmente a produtos organofosforados e piretroides (MiLIO et al., 1987; CoCHRAN, 1989; LeE et al., 1996). Por isso, o uso adequado e consciente dos inseticidas, além de diminuir os impactos à saúde do homem e ao ambiente, prolonga a vida útil dos produtos utilizados comercialmente.

O objetivo do trabalho foi avaliar a eficiência e efeito residual de inseticidas aplicados sobre azulejo (face lisa) e ardósia (rugosa) para o controle de $B$. germanica.

\section{MATERIAL E MÉTODOS}

O experimento foi realizado no laboratório do Departamento de Fitossanidade da Faculdade de Ciências Agrárias e Veterinárias - UNESP, Jaboticabal, SP, nos dias 3 e 4/5/2007, 18 e 19/5/2007, 4 e 5/6/2007,2e3/7/2007,1 e2/8/2007. A população de B. germanica utilizada foi criada a partir de setembro de 2006, proveniente de matrizes cedidas pelo Dr. Marcos Roberto Potenza, do Centro de Pesquisa e Desenvolvimento de Sanidade Vegetal do Instituto Biológico,São Paulo, caracterizadas como indivíduos suscetíveis ao tratamento com inseticidas.

As baratas foram criadas em sala não climatizada, dentro de caixas de plástico (56,1 L), cuja parte central da tampa foi substituída por tecido de algodão branco fixado com madeira e parafusos, possibilitando a circulação de ar. Para abrigo dos insetos, três a quatro bandejas de papelão, como as utilizadas para o comércio de ovos de galinha, foram dispostas horizontalmente no fundo da caixa de criação. Foram adaptados dois bebedouros (como os utilizados para pássaros) com algodão para controle da saída de água. O alimento foi fornecido numa placa de Petri de $7 \mathrm{~cm}$ de diâmetro (ração para gato triturada em liquidificador). Alimento e água foram fornecidos duas vezes por semana e as caixas de criação foram limpas uma vez por mês.

Os tratamentos utilizados foram (dosagens em produtos comerciais): lambdacialotrina (Demand 2,5 CS) $10 \mathrm{~mL} / \mathrm{L}$ de calda $\left(12,5 \mathrm{mg} / \mathrm{m}^{2}\right)$; lambdacialotrina (Icon 5,0 CE) $5 \mathrm{~mL} / \mathrm{L}$ de calda $(12,5 \mathrm{mg} /$ $\mathrm{m}^{2}$ ); deltametrina (Deltagard WG) 2 saches $/ 10 \mathrm{~L}$ de calda $\left(12,5 \mathrm{mg} / \mathrm{m}^{2}\right)$; lambdacialotrina (Zeon $50 \mathrm{CS}$ ) $5 \mathrm{~mL} / \mathrm{L}$ de calda $\left(12,5 \mathrm{mg} / \mathrm{m}^{2}\right)$; deltametrina (Deltek CS) $5 \mathrm{~mL} / \mathrm{L}$ de calda $\left(6,25 \mathrm{mg} / \mathrm{m}^{2}\right)$; diclorvos (DDVP $1000 \mathrm{CE}) 5 \mathrm{~mL} / \mathrm{L}$ de calda $\left(250 \mathrm{mg} / \mathrm{m}^{2}\right)$ e a testemunha. O delineamento experimental adotado foi o de blocos casualizados com quatro repetições. Como as repetições foram realizadas em dias diferentes, utilizou-se cada dia de repetição como um bloco do experimento.

Os inseticidas foram aplicados com um pulverizador pressurizado $\left(\mathrm{CO}_{2}\right)$ equipado com um bico de jato plano contínuo 8002E (modelo TP, marca TeeJet, Spraying Systems Co.), sob uma pressão de $350 \mathrm{kPa}\left(50,8 \mathrm{lbf} / \mathrm{pol}^{2}\right)$ e volume de aplicação de $40 \mathrm{~mL} / \mathrm{m}^{2}$.

Os inseticidas foram aplicados em placas de azulejo (face lisa) e placas de ardósia (face rugosa). Após o tratamento das superfícies esperou-se uma hora para a secagem das placas e, em seguida, foram liberadas 10 baratas adultas (cinco machos e cinco fêmeas) no centro da área que recebeu pulverização, para que os indivíduos caminhassem sobre a área tratada. Esta foi limitada por um pote de plástico invertido untado com vaselina nos bordos para que as baratas não fugissem.

Depois de confinadas por 15 minutos na área tratada, as baratas foram anestesiadas com $\mathrm{CO}_{2} \mathrm{e}$ colocadas em potes plásticos para avaliação. As avaliações da mortalidade foram realizadas por meio da observação direta dos indivíduos quanto ao efeito "knock-down" e 1, 2, 4, 24, 48 e 72 horas após a exposição (HAE). 
Para avaliação do efeito residual dos produtos aplicados, as placas tratadas foram guardadas em prateleiras. Com 30, 60 e 90 dias após a aplicação, 10 adultos (cinco machos e cinco fêmeas) foram colocados nas placas para verificar o período residual dos inseticidas.

Os dados obtidos foram transformados em arco seno $\sqrt{\mathrm{p} / 100}$ e submetidos à análise de variância e ao teste de Tukey $(\mathrm{p} \leq 0,05)$. Para cálculo da percentagem de eficiência de controle das baratas pelos inseticidas, os dados de mortalidade foram corrigidos em relação à testemunha, conforme a fórmula de Abbott (NAKANO et al., 1981).

\section{RESULTADOS E DISCUSSÕES}

Nas avaliações da mortalidade das baratas no dia da aplicação o efeito "knock-down" foi verificado para os inseticidas lambdacialotrina (2,5 CS) e deltametrina (WG), que não diferiram significativamente entre si (Tabela 1). As menores mortalidades foram do inseticida diclorvos que não diferiu significativa- mente da testemunha. Para as avaliações de quatro e 24 horas não houve diferença significativa entre os inseticidas lambdacialotrina (2,5 CS), lambdacialotrina $(5,0 \mathrm{CE})$ e deltametrina (WG), que resultaram em percentagens de eficiências superiores a $80 \%$ (Fig. 1). O inseticida lambdacialotrina (50 CS) também apresentou eficiência acima de $80 \%$ e diferiu significativamente da testemunha (Fig. 1). A menor mortalidade foi encontrada no inseticida diclorvos (Tabela 1). Para a avaliação de 48 HAE não houve diferença significativa entre lambdacialotrina $(2,5$ CS), lambdacialotrina (5,0 CE), deltametrina (WG) e lambdacialotrina (50 CS), que resultaram em eficiência maior que $80 \%$ (Fig. 1). A menor mortalidade foi para o diclorvos (Tabela 1). Para $72 \mathrm{HAE}$, os inseticidas não diferiram entre si, com exceção do diclorvos (Tabela 1), e apresentaram eficiência superior a $80 \%$. Estes resultados corroboram os relatados por POTENZA et al. (2003) que verificaram ser eficiente o inseticida lambdacialotrina, assim como os inseticidas betaciflutrina e clorpirifós, nas dosagens recomendadas pelo fabricante, até os 150 dias após a aplicação dos produtos.

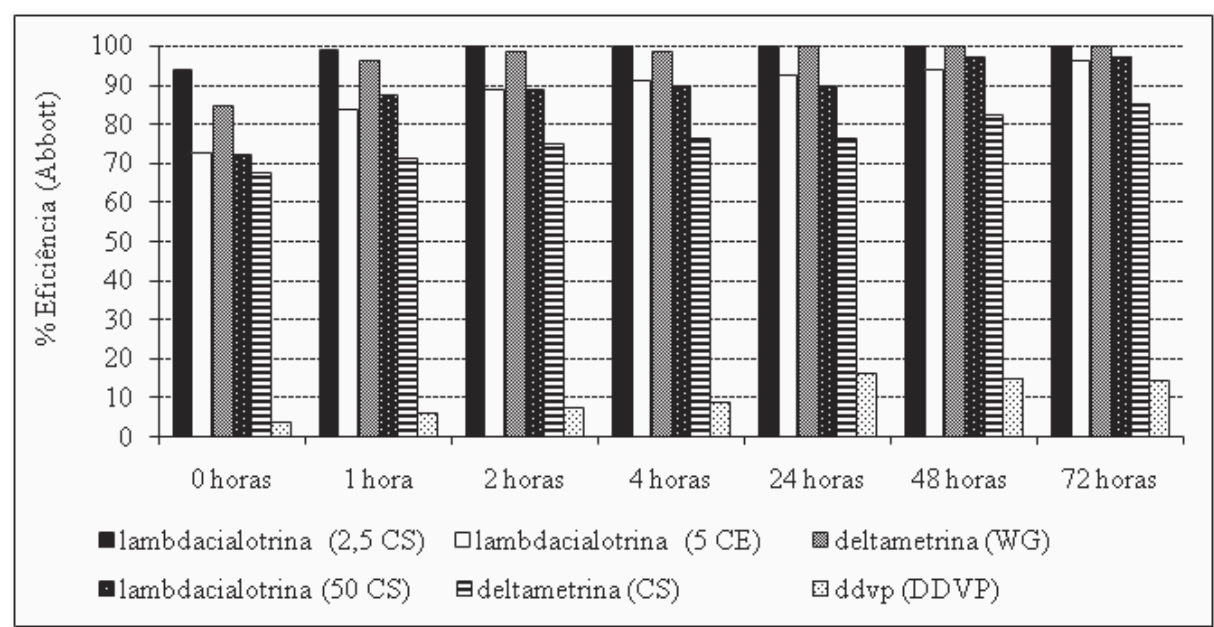

Fig. 1 - Eficiência (\%) dos inseticidas em Blattella germanica com aplicação em duas superfícies diferentes, no dia da aplicação. Jaboticabal, SP, 2007.

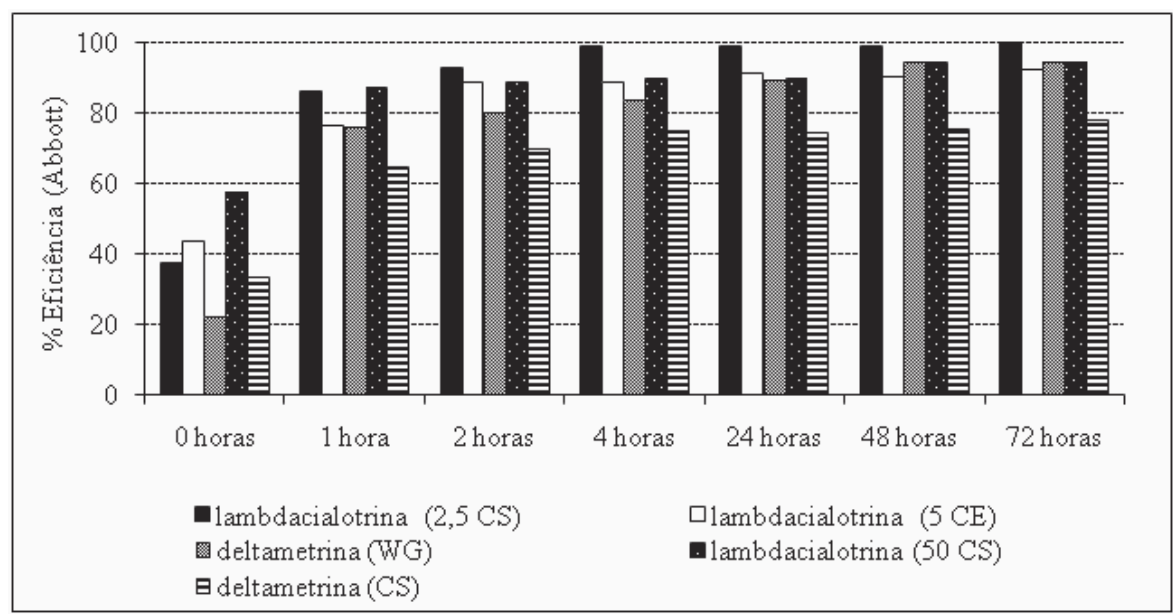

Fig. 2 - Eficiência (\%) dos inseticidas em Blattella germanica com aplicação em duas superfícies diferentes, 30 dias após a aplicação. Jaboticabal, SP, 2007. 
Tabela 1 - Mortalidade (\%)* de Blattella germanica sob efeito de inseticidas, em dois tipos de superfície, no dia da aplicação. Jaboticabal, SP, 2007.

\begin{tabular}{lccccccc}
\hline \multirow{2}{*}{ No dia da aplicação } & \multicolumn{5}{c}{ Mortalidade $^{1}$ (horas após exposição) } \\
\cline { 2 - 7 } & 0 & 1 & 2 & 4 & 24 & 48 & 72 \\
\hline Lisa & $50,45 \mathrm{a}$ & $55,28 \mathrm{a}$ & $57,78 \mathrm{a}$ & $58,36 \mathrm{a}$ & $58,65 \mathrm{a}$ & $63,56 \mathrm{a}$ & $65,10 \mathrm{a}$ \\
Rugosa & $44,71 \mathrm{~b}$ & $52,83 \mathrm{a}$ & $55,27 \mathrm{a}$ & $56,49 \mathrm{a}$ & $58,56 \mathrm{a}$ & $61,06 \mathrm{a}$ & $64,03 \mathrm{a}$ \\
\hline Teste F & $4,34^{*}$ & $1,37^{\mathrm{NS}}$ & $1,17^{\mathrm{NS}}$ & $0,50^{\mathrm{NS}}$ & $0,00^{\mathrm{NS}}$ & $0,76^{\mathrm{NS}}$ & $0,12^{\mathrm{NS}}$ \\
DMS (5\%) & 5,57 & 4,22 & 4,68 & 5,35 & 5,50 & 5,80 & 6,11 \\
\hline Lambdacialotrina (2,5 CS) & $78,14 \mathrm{a}$ & $86,90 \mathrm{a}$ & $89,09 \mathrm{a}$ & $89,09 \mathrm{a}$ & $89,09 \mathrm{a}$ & $89,09 \mathrm{a}$ & $89,09 \mathrm{a}$ \\
Lambdacialotrina (5,0 CE) & $59,17 \mathrm{~b}$ & $66,67 \mathrm{bc}$ & $72,90 \mathrm{~b}$ & $74,93 \mathrm{abc}$ & $75,95 \mathrm{ab}$ & $78,14 \mathrm{ab}$ & $82,52 \mathrm{a}$ \\
Deltametrina (WG) & $71,95 \mathrm{ab}$ & $85,05 \mathrm{a}$ & $86,90 \mathrm{a}$ & $86,90 \mathrm{ab}$ & $89,09 \mathrm{a}$ & $89,09 \mathrm{a}$ & $89,09 \mathrm{a}$ \\
Lambdacialotrina (50 CS) & $60,56 \mathrm{~b}$ & $70,89 \mathrm{~b}$ & $71,72 \mathrm{~b}$ & $72,74 \mathrm{bc}$ & $72,74 \mathrm{~b}$ & $85,89 \mathrm{ab}$ & $85,89 \mathrm{a}$ \\
Deltametrina (CS) & $56,03 \mathrm{~b}$ & $58,63 \mathrm{c}$ & $62,67 \mathrm{~b}$ & $64,86 \mathrm{c}$ & $64,86 \mathrm{~b}$ & $70,34 \mathrm{~b}$ & $74,04 \mathrm{a}$ \\
Diclorvos (DDVP) & $6,30 \mathrm{c}$ & $9,33 \mathrm{~d}$ & $11,52 \mathrm{c}$ & $12,53 \mathrm{~d}$ & $17,90 \mathrm{c}$ & $18,34 \mathrm{c}$ & $20,68 \mathrm{~b}$ \\
Testemunha & $0,91 \mathrm{c}$ & $0,91 \mathrm{~d}$ & $0,91 \mathrm{c}$ & $0,91 \mathrm{~d}$ & $0,91 \mathrm{~d}$ & $5,28 \mathrm{c}$ & $10,69 \mathrm{~b}$ \\
\hline Teste F & $72,72^{* *}$ & $160,36^{* *}$ & $135,89^{* *}$ & $104,73^{* *}$ & $95,70^{* *}$ & $87,00^{* *}$ & $72,11^{* *}$ \\
DMS (5\%) & 16,00 & 12,13 & 13,45 & 15,36 & 15,79 & 16,65 & 17,55 \\
\hline
\end{tabular}

*Dados transformados em arco seno $\sqrt{\mathrm{p} / 100}$.

${ }^{1}$ Médias seguidas pela mesma letra na coluna não diferem entre si pelo teste de Tukey, no nível de 5\% de probabilidade.

Tabela 2 - Mortalidade (\%)* de Blattella germanica sob efeito de inseticidas, em dois tipos de superfície, 30 dias após a aplicação. Jaboticabal, SP, 2007.

\begin{tabular}{llllllll}
\hline \multirow{2}{*}{30 dias após aplicação } & \multicolumn{7}{l}{ Mortalidade $^{1}$ (horas após exposição) } \\
\cline { 2 - 7 } & 0 & 1 & 2 & 4 & 24 & 48 & 72 \\
\hline Lisa & $49,26 \mathrm{a}$ & $64,05 \mathrm{a}$ & $67,48 \mathrm{a}$ & $70,01 \mathrm{a}$ & $70,01 \mathrm{a}$ & $74,73 \mathrm{a}$ & $75,01 \mathrm{a}$ \\
Rugosa & $10,96 \mathrm{~b}$ & $45,51 \mathrm{~b}$ & $50,24 \mathrm{~b}$ & $54,67 \mathrm{~b}$ & $54,67 \mathrm{~b}$ & $61,42 \mathrm{~b}$ & $64,37 \mathrm{~b}$ \\
\hline Teste F & $166,92^{* *}$ & $53,35^{* *}$ & $63,76^{* *}$ & $29,83^{* *}$ & $29,83^{* *}$ & $17,58^{* *}$ & $11,72^{* *}$ \\
DMS (5\%) & 6,03 & 5,17 & 4,40 & 5,72 & 5,72 & 6,42 & 6,33 \\
\hline Lambdacialotrina (2,5 CS) & $30,69 \mathrm{bc}$ & $71,05 \mathrm{a}$ & $77,12 \mathrm{a}$ & $86,90 \mathrm{a}$ & $86,90 \mathrm{a}$ & $86,90 \mathrm{a}$ & $89,09 \mathrm{a}$ \\
Lambdacialotrina (5,0 CE) & $40,55 \mathrm{ab}$ & $66,14 \mathrm{ab}$ & $77,06 \mathrm{a}$ & $77,06 \mathrm{ab}$ & $77,06 \mathrm{ab}$ & $78,64 \mathrm{ab}$ & $81,85 \mathrm{ab}$ \\
Deltametrina (WG) & $24,42 \mathrm{c}$ & $61,50 \mathrm{ab}$ & $64,35 \mathrm{bc}$ & $68,40 \mathrm{~b}$ & $68,40 \mathrm{~b}$ & $85,06 \mathrm{a}$ & $85,06 \mathrm{a}$ \\
Lambdacialotrina (50 CS) & $55,80 \mathrm{a}$ & $74,60 \mathrm{a}$ & $75,43 \mathrm{ab}$ & $76,26 \mathrm{ab}$ & $76,26 \mathrm{ab}$ & $81,50 \mathrm{ab}$ & $81,50 \mathrm{ab}$ \\
Deltametrina (CS) & $28,28 \mathrm{bc}$ & $54,48 \mathrm{~b}$ & $58,28 \mathrm{c}$ & $64,51 \mathrm{~b}$ & $64,51 \mathrm{~b}$ & $66,02 \mathrm{~b}$ & $67,28 \mathrm{~b}$ \\
Testemunha & $0,91 \mathrm{c}$ & $0,91 \mathrm{c}$ & $0,91 \mathrm{~d}$ & $0,91 \mathrm{c}$ & $0,91 \mathrm{c}$ & $10,34 \mathrm{c}$ & $13,36 \mathrm{c}$ \\
\hline Teste F & $25,15^{* *}$ & 77,26 & $123,73^{* *}$ & $81,58^{* *}$ & $81,58^{* *}$ & $56,48^{* *}$ & $56,29^{* *}$ \\
DMS (5\%) & 15,52 & 13,29 & 11,30 & 14,71 & 14,71 & 16,62 & 16,27 \\
\hline
\end{tabular}

*Dados transformados em arco seno $\sqrt{\mathrm{p} / 100}$.

${ }^{1}$ Médias seguidas pela mesma letra na coluna, não diferem entre si pelo teste de Tukey, ao nível de $5 \%$ de probabilidade.

Em relação ao tipo de superfície no dia da aplicação, o maior efeito "knock-down" nas baratas foi verificado na superfícielisa; para os demais horários de avaliação não houve diferença significativa entre os tipos de superfície (Tabela 1).

Aos 30 dias após a aplicação dos inseticidas, o inseticida diclorvos não apresentava mais nenhuma eficiência e, desse modo, não foi mais apresentado nas tabelas de resultados. Nesta data, o maior efeito "knock-down" foi verificado para o lambdacialotrina (50 CS) que não diferiu significativamente do lambdacialotrina (5,0 CE) (Tabela 2). Entretanto, nenhum inseticida apresentou eficiência superior a $80 \%$ (Fig. 2). Na avaliação de uma HAE, a maior mortalidade foi verificada para o lambdacialotrina (50 CS), porém não diferiu dos inseticidas lambdacialotrina (2,5 CS), lambdacialotrina (5,0 CE) e deltametrina (WG). Para as avaliações de 2, 4 e
$24 \mathrm{HAE}$, as maiores mortalidades foram verificadas para os inseticidas lambdacialotrina $(2,5 \mathrm{CS})$, lambdacialotrina (50 CS), lambdacialotrina (5,0 CE) e deltametrina (WG), que apresentaram eficiência superior a $80 \%$ (Fig. 2). Para os horários de 48 e 72 HAE, as maiores mortalidades foram verificadas para o inseticida lambdacialotrina (2,5 CS) que não diferiu do lambdacialotrina $(5,0 \mathrm{CE})$, deltametrina (WG) e lambdacialotrina (50 CS) (Tabela 2). As eficiências superaram os $80 \%$ para os inseticidas anteriormente citados (Fig. 2). Em relação ao tipo de superfície para todas as avaliações, durante as exposições das baratas, as maiores mortalidades foram observadas para a superfície lisa (Tabela 2). Esses resultados corroboram com os relatados por SALMERON; OMOTо (2004) que obtiveram controle da população suscetível de $B$. germanica utilizando deltametrina $(12,5 \mathrm{mg})$, na superfície lisa. 
Tabela 3 - Mortalidade (\%)* de Blattella germanica sob efeito de inseticidas, em dois tipos de superfície, 60 dias após a aplicação. Jaboticabal, SP, 2007.

\begin{tabular}{lccccccc}
\hline \multirow{2}{*}{60 dias após aplicação } & \multicolumn{7}{c}{ Mortalidade $^{1}$ (horas após exposição) } \\
\cline { 2 - 7 } & 0 & 1 & 2 & 4 & 24 & 48 & 72 \\
\hline Lisa & $70,40 \mathrm{a}$ & $71,87 \mathrm{a}$ & $72,93 \mathrm{a}$ & $73,67 \mathrm{a}$ & $74,40 \mathrm{a}$ & $74,40 \mathrm{a}$ & $77,26 \mathrm{a}$ \\
Rugosa & $35,21 \mathrm{~b}$ & $49,71 \mathrm{~b}$ & $50,44 \mathrm{~b}$ & $51,78 \mathrm{~b}$ & $51,78 \mathrm{~b}$ & $58,93 \mathrm{~b}$ & $62,91 \mathrm{~b}$ \\
\hline Teste F & $252,47^{* *}$ & $33,27^{* *}$ & $32,18^{* *}$ & $28,73^{* *}$ & $31,82^{* *}$ & $18,40^{* *}$ & $14,73^{* *}$ \\
DMS (5\%) & 4,51 & 7,82 & 8,07 & 8,31 & 8,16 & 7,34 & 7,61 \\
\hline Lambdacialotrina (2,5 CS) & $68,63 \mathrm{a}$ & $79,66 \mathrm{a}$ & $81,85 \mathrm{a}$ & $82,68 \mathrm{a}$ & $82,68 \mathrm{a}$ & $82,68 \mathrm{a}$ & $83,70 \mathrm{a}$ \\
Lambdacialotrina (5,0 CE) & $63,12 \mathrm{a}$ & $71,77 \mathrm{a}$ & $74,98 \mathrm{a}$ & $74,98 \mathrm{a}$ & $77,17 \mathrm{a}$ & $82,52 \mathrm{a}$ & $82,52 \mathrm{a}$ \\
Deltametrina (WG) & $59,38 \mathrm{a}$ & $72,67 \mathrm{a}$ & $72,67 \mathrm{a}$ & $78,07 \mathrm{a}$ & $78,07 \mathrm{a}$ & $79,92 \mathrm{a}$ & $80,68 \mathrm{a}$ \\
Lambdacialotrina (50 CS) & $63,27 \mathrm{a}$ & $74,86 \mathrm{a}$ & $74,86 \mathrm{a}$ & $74,86 \mathrm{a}$ & $74,86 \mathrm{a}$ & $78,07 \mathrm{a}$ & $81,02 \mathrm{a}$ \\
Deltametrina (CS) & $61,53 \mathrm{a}$ & $64,85 \mathrm{a}$ & $64,85 \mathrm{a}$ & $64,85 \mathrm{a}$ & $64,85 \mathrm{a}$ & $69,65 \mathrm{a}$ & $73,68 \mathrm{a}$ \\
Testemunha & $0,91 \mathrm{~b}$ & $0,91 \mathrm{~b}$ & $0,91 \mathrm{~b}$ & $0,91 \mathrm{~b}$ & $0,91 \mathrm{~b}$ & $7,13 \mathrm{~b}$ & $18,94 \mathrm{~b}$ \\
\hline Teste F & $89,10^{* *}$ & $39,92^{* *}$ & $38,84^{* *}$ & $38,05^{* *}$ & $39,95^{* *}$ & $44,78^{* *}$ & $30,51^{* *}$ \\
DMS (5\%) & 11,60 & 20,11 & 20,76 & 21,37 & 20,99 & 18,88 & 19,57 \\
\hline
\end{tabular}

*Dados transformados em arco seno $\sqrt{\mathrm{p} / 100}$.

${ }^{1}$ Médias seguidas pela mesma letra nả coluna não diferem entre si pelo teste de Tukey, no nível de $5 \%$ de probabilidade.

Tabela 4 - Mortalidade (\%)* de Blattella germanica sob efeito de inseticidas, em dois tipos de superfície, 90 dias após a aplicação. Jaboticabal, SP, 2007.

\begin{tabular}{lccccccc}
\hline \multirow{2}{*}{90 dias após aplicação } & \multicolumn{5}{c}{ Mortalidade ${ }^{1}$ (horas após exposição) } \\
\cline { 2 - 7 } & 0 & 1 & 2 & 4 & 24 & 48 & 72 \\
\hline Lisa & $70,40 \mathrm{a}$ & $71,87 \mathrm{a}$ & $72,93 \mathrm{a}$ & $73,67 \mathrm{a}$ & $74,40 \mathrm{a}$ & $74,40 \mathrm{a}$ & $77,26 \mathrm{a}$ \\
Rugosa & $35,21 \mathrm{~b}$ & $49,71 \mathrm{~b}$ & $50,44 \mathrm{~b}$ & $51,78 \mathrm{~b}$ & $51,78 \mathrm{~b}$ & $58,93 \mathrm{~b}$ & $62,91 \mathrm{~b}$ \\
Teste F & $252,47^{* *}$ & $33,27^{* *}$ & $32,18^{* *}$ & $28,73^{* *}$ & $31,82^{* *}$ & $18,40^{* *}$ & $14,73^{* *}$ \\
DMS (5\%) & 4,51 & 7,82 & 8,07 & 8,31 & 8,16 & 7,34 & 7,61 \\
Lambdacialotrina (2,5 CS) & $68,63 \mathrm{a}$ & $79,66 \mathrm{a}$ & $81,85 \mathrm{a}$ & $82,68 \mathrm{a}$ & $82,68 \mathrm{a}$ & $82,68 \mathrm{a}$ & $83,70 \mathrm{a}$ \\
Lambdacialotrina (5,0 CE) & $63,12 \mathrm{a}$ & $71,77 \mathrm{a}$ & $74,98 \mathrm{a}$ & $74,98 \mathrm{a}$ & $77,17 \mathrm{a}$ & $82,52 \mathrm{a}$ & $82,52 \mathrm{a}$ \\
Deltametrina (WG) & $59,38 \mathrm{a}$ & $72,67 \mathrm{a}$ & $72,67 \mathrm{a}$ & $78,07 \mathrm{a}$ & $78,07 \mathrm{a}$ & $79,92 \mathrm{a}$ & $80,68 \mathrm{a}$ \\
Lambdacialotrina (50 CS) & $63,27 \mathrm{a}$ & $74,86 \mathrm{a}$ & $74,86 \mathrm{a}$ & $74,86 \mathrm{a}$ & $74,86 \mathrm{a}$ & $78,07 \mathrm{a}$ & $81,02 \mathrm{a}$ \\
Deltametrina (CS) & $61,53 \mathrm{a}$ & $64,85 \mathrm{a}$ & $64,85 \mathrm{a}$ & $64,85 \mathrm{a}$ & $64,85 \mathrm{a}$ & $69,65 \mathrm{a}$ & $73,68 \mathrm{a}$ \\
Testemunha & $0,91 \mathrm{~b}$ & $0,91 \mathrm{~b}$ & $0,91 \mathrm{~b}$ & $0,91 \mathrm{~b}$ & $0,91 \mathrm{~b}$ & $7,13 \mathrm{~b}$ & $18,94 \mathrm{~b}$ \\
\hline Teste F & $89,10^{* *}$ & $39,92^{* *}$ & $38,84^{* *}$ & $38,05^{* *}$ & $39,95^{* *}$ & $44,78^{* *}$ & $30,51^{* *}$ \\
\hline DMS (5\%) & 11,60 & 20,11 & 20,76 & 21,37 & 20,99 & 18,88 & 19,57 \\
\hline
\end{tabular}

*Dados transformados em arco seno $\sqrt{\mathrm{p} / 100}$.

${ }^{1}$ Médias seguidas pela mesma letra nả coluna não diferem entre si pelo teste de Tukey, no nível de $5 \%$ de probabilidade.

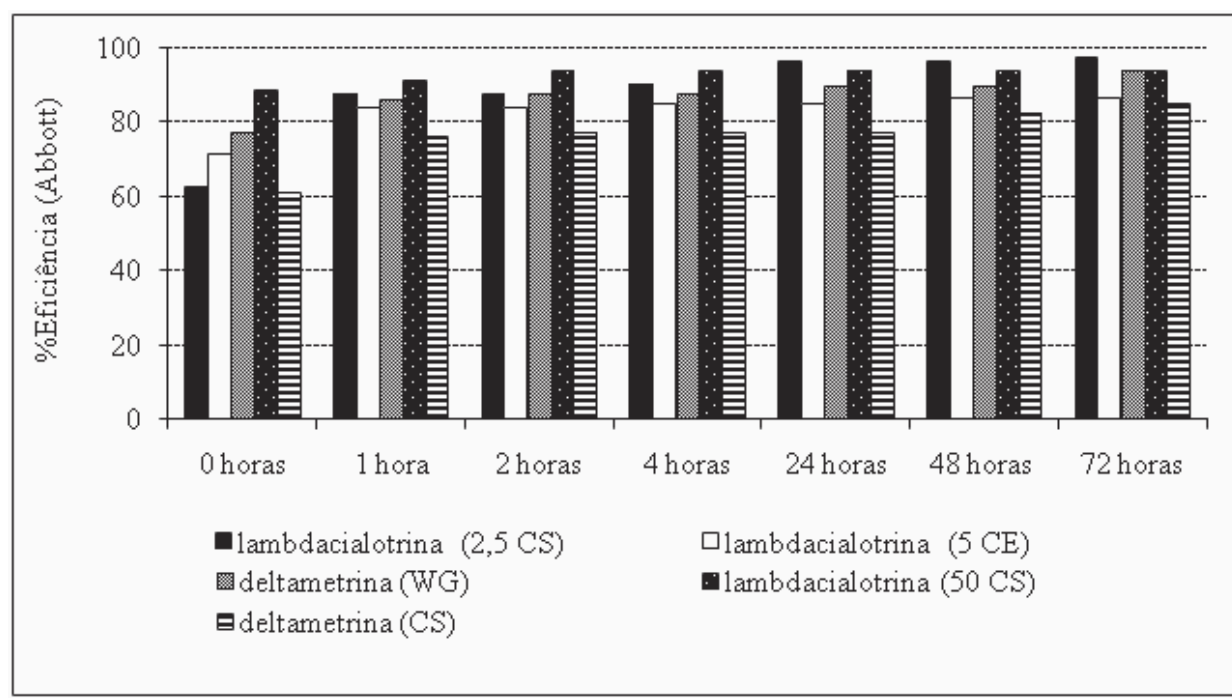

Fig. 3 - Eficiência (\%) dos inseticidas em Blattella germanica com aplicação em duas superfícies diferentes, 60 dias após a aplicação. Jaboticabal, SP, 2007. 


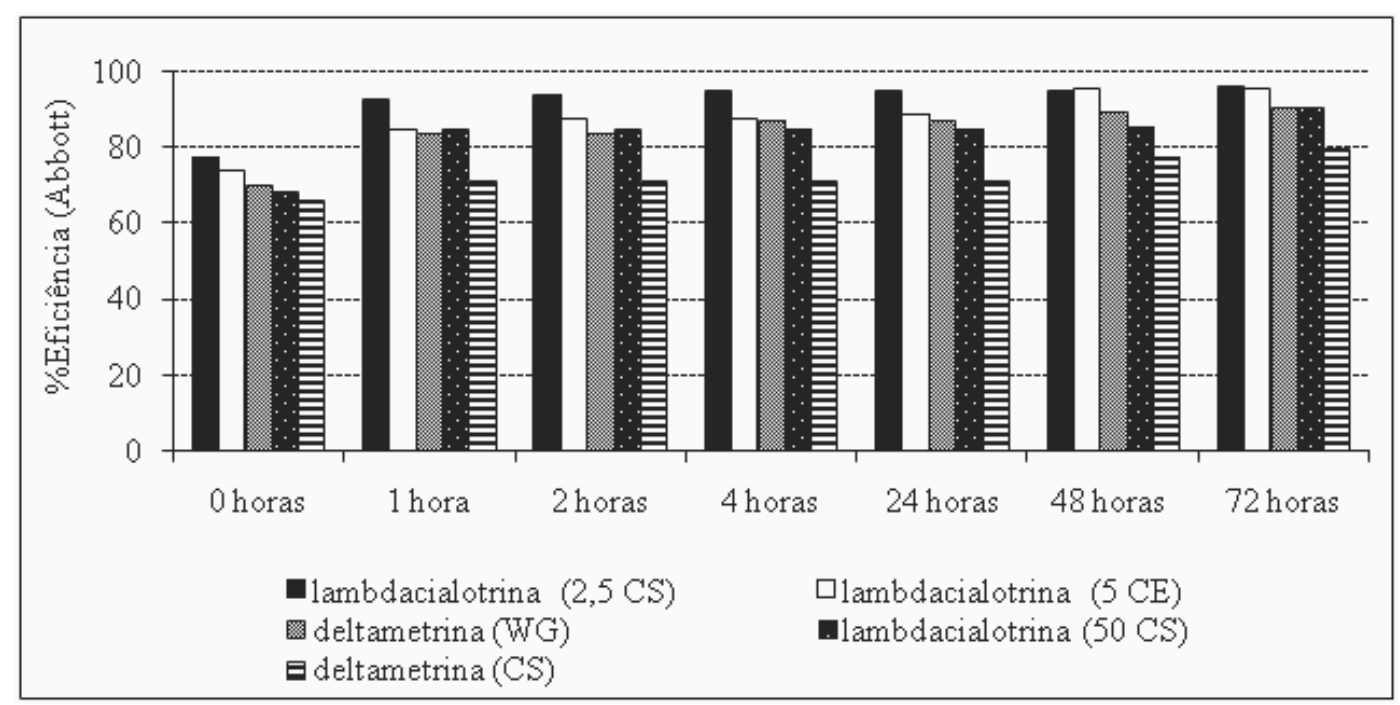

Fig. 4 - Eficiência (\%) dos inseticidas em Blattella germanica com aplicação em duas superfícies diferentes, 90 dias após a aplicação. Jaboticabal, SP, 2007.

Com 60 dias após a aplicação, as avaliações da mortalidade das baratas as 0,1, 2, 4, 24, 48 e72HAEnas áreas tratadas não diferiram significativamente entre os inseticidas, apresentando diferenças significativas apenas com a testemunha (Tabela 3). Nas avaliações de 1, 2, 4 e $24 \mathrm{HAE}$, os inseticidas lambdacialotrina (2,5CS), lambdacialotrina (5,0 CE), deltametrina (WG) e lambdacialotrina (50 CS) resultaram em eficiências superiores a 80\% (Fig. 3). Ao deixar as baratas expostas por 48 e $72 \mathrm{HAE}$ também o inseticida deltametrina (CS) apresentou eficiência superior a $80 \%$, assim como os demais inseticidas. Este aumento de eficiência dos inseticidas em relação ao tempo de exposição também foi constatado por PARREIRA et al. (2010). Em relação ao tipo de superfície, para todos os horários em que os adultos de B. germanica ficaram expostos aos inseticidas, as maiores mortalidades foram observadas na superfície lisa (Tabela 3).

Para todos os tempos de avaliação após exposição das baratas na área tratada, 90 dias após a aplicação, não houve diferença significativa entre os inseticidas, mas estes diferiram significativamente da testemunha (Tabela 4). Nas avaliações de 1, 2, 4, 24, 48 e $72 \mathrm{HAE}$ das baratas nas superfícies tratadas, os inseticidas lambdacialotrina (2,5CS), lambdacialotrina (5,0 CE), deltametrina (WG) e lambdacialotrina (50 CS) resultaram em eficiências superiores a $80 \%$ de controle (Fig. 4). Em relação aos tipos de superfície para todos os horários de avaliação, as maiores mortalidades de baratas foram observadas para a superfície lisa (Tabela 4).

\section{CONCLUSÕES}

- No dia da aplicação dos inseticidas não ocorre diferença entre as superfícies no controle de $B$.germanica;
- O controle de B. germanica é mais eficiente em superfície lisa (azulejo) quando comparado com superfície rugosa (ardósia), no período de 30 a 90 dias após a aplicação;

- Os inseticidas, nas dosagens utilizadas, lambdacialotrina (2,5 CS), lambdacialotrina $(5,0 \mathrm{CE})$, lambdacialotrina (50 CS) e deltametrina (WG) proporcionam excelente controle de B. germanica até 90 dias após a aplicação;

-Oinseticida deltametrina (CS), na dose utilizada, apresenta baixa eficiência, enquantoqueodiclorvos(DDVP) éconsiderado ineficientepara ocontrolede B. germanica.

\section{REFERENCIAS}

APPEL, A.G. Blattella and related species. In: (Ed.). Understanding and a controlling

the Germam cockroach. New York: Oxford University Press, 1995. p.1-19. cap. 1.

BRANESS, G.A. Residual effectiveness of insecticides for control of German cockroach (Dictyoptera: Blattellidae) in food-handling establishments. Journal of Economic Entomology, v.83, n.3, p.1907-1911, 1990.

BRANESS, G.A.; COSTER, D.C.; BENNETT, G.W. Logistic models describing effects of temperature and humidity on residual effectiveness of chlorpyrifos and cyfluthrin formulations against German cockroaches (Dictyoptera: Blattellidae). Journal of Economic Entomology, v.84, n.6, p.1746-1752, 1991.

COCHRAN, D.G. Monitoring for insecticide resistance in field-collected "strains" of the German cockroach (Dictyoptera: Blattellidae). Journal of Economic Entomology, v.82, n.2, p.336-341, 1989. 
CORNWELL, P.B. The Cockroach: A laboratory insect and an industrial pest. London: The Rentokil Library, 1968. v.1, 391p.

EL-AWAMI, I.O.; DENT, D.R. The interaction of surface and dust particle size on the picy-up and grooming of the German cockroach Blattella germanica. Entomologia Experimentais et Applicata, v.77, n.1, p.81-87, 1995.

GALLO, D.; NAKANO, O.; SILVEIRA NETO, S.; CARVALHO, R.P.L.; BAPTISTA, G.C. de; BERTI FILHO, E.; PARRA, J.R.P.; ZUCCHI, R.A.; ALVES, S.B.; VENDRAMIM, J.D.; MARCHINI, L.C.; LOPES, J.R.S.; OMOTO, C. Entomologia agrícola. Piracicaba: FEALQ, 2002. 920p.

KAAKEH, W.; REID, B.L.; KAAKEH, N.; BENNETT, G.W. Rate determination, indirect toxicity, contact activity, and residual persistance of lufenuron for the control of the German cockroach (Dictyoptera: Blattellidae). Journal of Economic Entomology, v.90, n.2, p.510-522, 1997.

LEE, C.Y.; YAP, H.H.; CHONG, N.L. Insecticide resistance and sinergism in field collected German cockroaches (Dictyoptera: Blattellidae) in Peninsular Malaysia. Bulletin of Entomological Research, v.86, p.675682, 1996.

MILIO, J.F.; P.G. KOEHLER P.G.; PATTERSON R.S. Evaluation of three methods for detecting chlorpyrifos resistance in German cockroach (Orthoptera: Blattellidae) populations. Journal of Economic Entomology, v.80, p.44-46, 1987.

NAKANO, O.; SILVEIRA NETO, S.; ZUCCHI, R.A. Entomologia econômica. São Paulo: Editora Ceres, 1981. 314p.
PÉREZ, J.R. La cucaracha como vetor de agentes patógenos. Boletin de la Oficina Sanitaria Panamericana, v.107, n.1, p.41-53, 1989.

PARREIRA, R.S.; FERREIRA, M.C.; MARTINELLI, N.M.; SILVA, I.C.; FERNANDES, A.P.; ROMANI, G.N. Mortalidade de Blattella germanica (L., 1767) (Blattodea: Blattellidae) sob diferentes áreas e períodos de exposição a diferentes inseticidas Bioscience Journal, v.26, n.1, p.40-51, 2010

POTENZA, M.R.; SANTOS, J.M.F.; SILVA, R.S.; ALVES, J.N. Avaliação de diferentes pontas de Pulverização na eficácia de inseticidas no tratamento de superfície para o controle de Blattella germanica (Dictyoptera: Blattellidae). Arquivos do Instituto Biológico. São Paulo, v.70, n.3, p.355-358, 2003.

ROSS, M.H. Response of behaviorally resistant German cockroaches (Dictyoptera: Blattellidae) to the active ingredient in a commercial bait. Journal of Economic Entomology, v.91, n.1, p.150-152, 1998.

SALMERON, E.; OMOTO, C. Mistura de deltametrina e clorpirifós no manejo da resistência de Blattella germanica (Linnaeus, 1757) (Dictyoptera: Blattellidae) a deltametrina. Entomotropica, v.19, n.2, p.85-89, 2004.

SMITH II, L.M.; APPEL, A.G.; MACK, T.P.; KEEVER, G.J. Comparison of traps and development of a two-stage sampling plan for smokybrown cockroaches (Dictyoptera: Blattellidae). Journal of Economic Entomology, v.90, n.5, p.1222-1231, 1997.

Recebido em 24/5/10

Aceito em 16/5/11 